A Dynamic Neural Field Model of Temporal Order Judgments

Lauren N. Hecht ${ }^{1}$, John P. Spencer ${ }^{2}$, \& Shaun P. Vecera ${ }^{2}$

${ }^{1}$-Gustavus Adolphus College, ${ }^{2}$-University of Iowa

Corresponding Author:

Lauren N. Hecht

Gustavus Adolphus College

Department of Psychological Science

800 West College Ave

Saint Peter, MN 56082

E-mail: lhecht@gustavus.edu

WORD COUNT: 7,409 


\begin{abstract}
Temporal ordering of events is biased, or influenced, by perceptual organization - figure-ground organization - and by spatial attention. For example, within a region assigned figural status or at an attended location, onset events are processed earlier (Shore et al., 2001; Lester et al., 2009), and offset events are processed for longer durations (Hecht \& Vecera, 2011; Rolke et al., 2006). Here, we present an extension of a dynamic field model of change detection (Johnson, Spencer, Luck, \& Schöner, 2009; Johnson, Spencer, \& Schöner, 2009) that accounts for both the onset and offset performance for figural and attended regions. The model posits that neural populations processing the figure are more active, resulting in a peak of activation that quickly builds toward a detection threshold when the onset of a target is presented. This same enhanced activation for some neural populations is maintained when a present target is removed, creating delays in the perception of the target's offset. We discuss the broader implications of this model, including insights regarding how neural activation can be generated in response to the disappearance of information.
\end{abstract}

Keywords: spatial attention, perceptual organization, figure-ground organization, temporal order judgments, modeling, onsets, offsets 


\section{A Dynamic Neural Field Model of Temporal Order Judgments}

One heuristic commonly used to disambiguate information within a visual scene is figure-ground organization, whereby information is combined to form a single perceptual group (i.e., figure) that falls into the foreground while the surrounding, irrelevant information falls into the background (Palmer \& Rock, 1994). Image-based cues (e.g., Palmer, 1999, 2002; see also Pomerantz \& Kubovy, 1986, Rock, 1975, 1995, and Rubin, 1915/1958; Vecera, Vogel, \& Woodman, 2002), top-down processes (e.g., Baylis \& Driver, 1995; Driver \& Baylis, 1996; Julesz, 1984; Peterson, 1994, 1999; Peterson \& Gibson, 1991, 1993, 1994; Peterson, Harvey \& Weidenbacher, 1991; Rock, 1975; Vecera, Flevaris, \& Filapek, 2004; Vecera \& O’Reilly, 1998, 2000), and early perceptual processes, such as spatial and temporal perception (e.g., Klymenko \& Weisstein, 1986, 1989a, 1989b; Klymenko, Weisstein, Topolski \& Hsieh, 1989), influence figure-ground organization. However, recent research has also demonstrated that figure-ground organization impacts other perceptual processes, including shape discriminability (Wong \& Weissten, 1982, 1983; Lazareva et al., 2006), choice reaction time (Nelson \& Palmer, 2007), perceived contrast (Self et al., 2015), and temporal processing (Hecht \& Vecera, 2011; Lester, Hecht, \& Vecera, 2009). When making temporal order judgments (TOJs) regarding the onset of two targets, the target on the ground must appear before the target on the figure in order to perceive the events as occurring simultaneously, suggesting a perceptual processing advantage such that figures undergo perceptual processing prior to grounds - a prior entry effect (Lester et al., 2009). In contrast, targets removed (i.e., offsetting) within the figure are perceived as disappearing later than targets removed from the ground region (Hecht \& Vecera, 2011). To accurately perceive the offset of information, it must be removed earlier on the figure than on 
the ground, suggesting that figures are afforded extended durations of processing - a temporal extension effect.

Although empirical work has led to an understanding of the consequences of figureground organization for temporal processing, a mechanistic account for these effects has not been established. Similar behavioral effects have been reported in the attention literature (e.g., Hein, Rolke, \& Ulrich, 2006; Rolke, Ulrich, \& Bausenhart, 2006; Shore \& Spence, 2005; Shore, Spence, \& Klein, 2001; Yeshurun, 2004; Yeshurun \& Levy, 2003), where attended regions have the same effects as figures, and researchers have proposed accounts to explain these effects. Unfortunately, there are currently no unified accounts that explain the suite of effects observed in TOJ tasks; rather, different accounts have been proposed to explain the processes underlying prior entry effects and those underlying temporal extension results. Although it is possible that each effect may arise from separate mechanisms, we pursue a comprehensive account here. In particular, we present a computational model that captures both the prior entry and temporal extension effects and, in doing so, provides a neurally-grounded understanding of the processes underlying TOJs.

The starting point for our model is to think about TOJs as a type of change detection task. Change detection consists of comparing the current state of the perceptual world relative to some prior encoded or remembered state to detect whether a change occurred. In the experiments that first noted prior entry and temporal extension as consequences of figure-ground organization (Hecht \& Vecera, 2011; Lester et al., 2009), observers determined the temporal order in which two stimuli onset (or offset) within the display. To properly order these temporal events, the state of the visual scene had to be encoded at the start of the trial and compared to incoming 
perceptual information to detect a change in the onset or offset of information. Thus, the temporal ordering task falls into a broad class of tasks that probe change detection abilities.

Currently, there is only one model that provides an account of the neural processes that underlie change detection: the dynamic neural field (DNF) model proposed by Johnson, Spencer, and colleagues (Johnson, Spencer, Luck, \& Schöner, 2009; Johnson, Spencer, \& Schöner, 2009). This three-layer DNF model is shown in Figure 1. The perceptual field $(\mathrm{PF}(u))$ is an excitatory neural layer that is reciprocally coupled to an inhibitory layer $(\operatorname{Inhib}(v))$. There is also a second excitatory layer - the working memory field $(\mathrm{WM}(w))$ - that is reciprocally coupled to the inhibitory layer. Each layer is metrically organized according to a functional topography tuned to a particular stimulus dimension such as a continuous hue dimension or the spatial dimension of the retina. Within the excitatory layers, above-threshold $(>0)$ neural sites pass excitation to their neighbors and also stimulate associated sites in the inhibitory layer. The stimulated sites in the inhibitory layer then pass inhibition back to the excitatory layers via a Gaussian projection that spreads inhibition laterally (see Amari, 1977). Input from the visual world is fed into PF. WM also receives a weaker copy of this input. Stimulation can result in localized 'peaks' of activation which reflect, for instance, perceptually encoded stimuli in PF. PF then stimulates WM via a feed-forward connection to WM which helps consolidate peaks in WM. Note that excitatory and inhibitory interactions are stronger in WM than in PF. Consequently, peaks in PF quickly decay once a stimulus is removed, while peaks in WM can be actively maintained during memory delays due to stronger recurrent interactions.

To understand how this network detects changes, consider a simple version of a change detection task - detecting a shift in the location of a stimulus on the retina. To examine whether participants can detect such a change, we might display an initial target position. This stimulus 
input would build a peak of activation in PF. With an input of sufficient strength and duration, this peak in PF would also give rise to a peak in the WM layer. When the stimulus is turned 'off', the peak in PF would collapse, both because recurrent interactions in PF are weak and because PF receives inhibitory input from the peak that is maintained in WM (due to the shared inhibitory layer). If we present an item to the network at the same position, the inhibition in PF can suppress the formation of a peak. This is the basis of a 'same' decision in the network. If, by contrast, we present an item at a new location, a peak readily forms in PF and the system actively detects that a 'different' stimulus has been presented.

Conceptually, these two types of responses might form the basis of TOJs. In particular, if we consider the formation of a peak in PF as a type of perceptual decision, it is easy to see how this system could detect the onset of a stimulus. But how does a neural system detect the offset of information? This is trickier because offsets naturally lead to a reduction in activation, yet response generation requires the reverse - the active generation of a neural decision. Here we explore whether the interaction between PF and WM might give rise to offset detection. In particular, when a stimulus has been encoded in WM, the associated sites in PF are suppressed. If an offset event led to the loss of a peak in WM, this suppression would be removed and other sources of input — such as input from an attentional system monitoring the offset locationwould then be free to build a peak in PF and drive an offset response.

In the remainder of the paper, we examine whether the neural processes that underlie change detection in the DNF model can be extended to explain TOJs. We begin by describing how we embed the DNF model in the TOJ task, including the addition of a response field that generates neural decisions about the order of onsets and offsets. We then simulate data from Lester et al. (2009) and Hecht and Vecera (2011). We conclude by discussing the implications of 
the DNF model for TOJs and how this model compares to other efforts to explain prior entry and temporal extension effects in the attention literature.

\section{A DNF Model of Temporal Order Judgments}

In this section, we provide an overview of the DNF model, the simulation method used, and the simulation results. The goal was to examine whether the change detection model from Johnson et al. (2009) could be extended to capture the insight that temporal order judgments reflect a type of change detection decision. In addition, we explored whether a single, unified account could explain both prior entry and temporal extension effects. To preview our results, we demonstrate below that sensitivity to a change in the onset of information can be accomplished by monitoring activation dynamics within the perceptual field in the DNF model. Detecting a change in the offset of information, however, results from the interaction between the perceptual and working memory layers. Finally, a slight boost in activation associated with target locations on a 'figure' or at an attended location is sufficient to explain both prior entry and temporal extension effects within the same neurodynamic model.

Model Architecture

We used the three-layer architecture of the change detection model (for model equations, see Johnson, Spencer, \& Schöner, 2009; Johnson et al., 2009). We then added three inputs to the model to capture the TOJ task. We also added a response layer since the goal of the task was to identify which attended location appeared/disappeared first (rather than indicate 'same' or 'different'). Although the addition of the response layer makes the current model different from the Johnson et al.'s change detection model architecturally, the underlying processing dynamics used to explain both change detection and temporal order judgments are the same between the 
models. Thus, our modeling efforts suggest that a common mechanism might underlie both change detection performance and temporal order judgments.

Three sources of input were added to the model to capture the events on each trial: Segmentation (Seg), Task, and Target. Seg reflects the segmentation of the two regions present in the display. This reflects the type of input that might be received from a process of figureground organization (see, e.g., Roelfsema et al., 2002; Vecera \& O’Reilly, 1998, 2000). There were three types of Seg inputs to reflect the task conditions we examined: (1) figure and ground (when two regions abutted one another) with activation associated with the figure (i.e., activation at one location, $-30^{\circ}$, and not at the other, $30^{\circ}$ ), (2) separated figure and ground with activation at both locations due to ambiguity in the display, and (3) no input at both locations when the figure and ground were presented as separated regions. Examples of these types of displays appear in Figure 2; we have used such displays in our previous research (e.g., Hecht \& Vecera, 2011), and findings from those studies are simulated here.

The Task input highlighted the relevant target locations, which consistently occurred at the center of the figure and ground regions. Thus, because these locations were fixed, Task input was constant at both $-30^{\circ}$ and $30^{\circ}$. The final input was the Target input. The Target input grew and/or receded in time to reflect the onset and offset movements of the target. For onsets, input to PF grew in strength across $110 \mathrm{~ms}$, and then its strength died out across an additional $110 \mathrm{~ms}$. For offsets, input to PF started strong to reflect the initial presentation of the target, and its strength receded across $110 \mathrm{~ms}$ in accordance with the disappearance of the targets.

In addition to the inputs, we also added a new response field to the change detection model. In the change detection model, the system generated a same or different judgment on each trial (Johnson, Spencer, \& Schoner, 2009; Johnson et al., 2009). By contrast, in the TOJ 
task, the model must provide a localized response to indicate which target onset (or offset) first. Thus, we added a response field that was reciprocally coupled to PF but was selective in its response; that is, based on input received from $\mathrm{PF}$, this field would build a peak at a left $(-30)$ or right (30) location indicating which location was associated with the first target onset or offset. Note that the response field had strong global inhibition, making this field operate as a 'winnertake-all' field (see $k_{r_{-} i n h i b}$ in Table 1). This decision mechanism was realized by the equation:

$$
\begin{aligned}
& \tau \dot{r}(x, t)=-r(x, t)+h_{r}+q_{h} \xi(t)+\int c_{r_{-} e x c i t e}\left(x-x^{\prime}\right) \Lambda_{r_{-} e x c i t e}\left(r\left(x^{\prime}, t\right)\right) d x^{\prime} \\
& -\int c_{r_{-} i n h i b}\left(x-x^{\prime}\right) \Lambda_{r_{-} i n h i b}\left(r\left(x^{\prime}, t\right)\right) d x^{\prime}+\int c_{r u}\left(x-x^{\prime}\right) \Lambda_{r u}\left(u\left(x^{\prime}, t\right)\right) d x^{\prime} \\
& +T_{r}(x, t)+q \int c_{n}\left(x-x^{\prime}\right) \xi\left(x^{\prime}, t\right) d x^{\prime}
\end{aligned}
$$

where $\dot{r}(x, t)$ is the rate of change of the activation level for each neuron across the spatial dimension, $x$, as a function of time, $t$. The first factor that contributes to the rate of change of activation in the response layer is the current activation in the field, $-r(x, t)$. This component is negative so that activation changes in the direction of the resting level, $\mathrm{h}_{\mathrm{r}}$. Next, activation is influenced by noise in the resting level as well as by local excitation and lateral inhibition. The next term in the equation is input from $\operatorname{PF}(u)$. Finally, the response layer receives activation from the target input as well as spatially correlated noise (for details, see Johnson et al., 2009).

The final modification to the change detection model was the addition of coupling between the response layer and PF. In particular, the response layer fed back onto PF according to the modified equation (1) taken from Johnson, Spencer, Luck, et al. (2009):

$$
\tau \dot{u}(x, t)=-u(x, t)+h_{u}+\ldots \int c_{u r}\left(x-x^{\prime}\right) \Lambda_{u r}\left(r\left(x^{\prime}, t\right)\right) d x^{\prime}
$$

Modeling Onsets: An Example 
An example of an onset simulation is presented in Figure 3. At the start of the trial, the TOJ model was provided $50 \mathrm{~ms}$ to relax into a stable state. As task-relevant information (e.g., figure-ground display) is presented, these events are input to the three-layer model for $500 \mathrm{~ms}$. In particular, Seg and Task inputs projected activation to the PF and WM fields, building activation at the corresponding spatial location of the two regions (e.g., figure and ground). As shown in Figure $3 \mathrm{~B}$, the activation of the peak building at the location of the figure (left) was stronger than at the ground location (right) due to the stronger Seg input for the figure. Note, however, that these inputs were relatively weak; thus, activation in PF remained below threshold.

After $550 \mathrm{~ms}$, the target events were introduced to the model via the Target input. The target inputs were presented at varying stimulus onset asynchronies (SOAs: 26, 50, 100 and 150 ms), following those from previous research (e.g., Lester et al., 2009). As the first target began to onset, the strength of the input at this location (left) began increasing to its full strength over the course of $110 \mathrm{~ms}$. Upon reaching full strength, the Target input began decreasing in strength over an additional $110 \mathrm{~ms}$. After the designated SOA, the second target location received the same growth and recession of activation.

As can be seen in the figure, the convergence of the Target, Seg, and Task inputs built a peak of activation in PF (i.e., activation > 0). Once a peak pierced the threshold in PF, activation began projecting more strongly into the response field at the location of this peak ("left" in Figure $3 \mathrm{C}$ ). As a consequence, activation at the left location in the response field raised above threshold generating a 'left' response. In this example, the figure was on the left; thus, the figural input and the temporal input cooperated to generate a decision that the 'figure' target led the 'ground' target. By varying the location (figure versus ground) of the leading stimulus and the SOAs between the target inputs, we can simulate psychophysical curves that allow us to compute 
the points of subjective equality for leading targets that appear on different regions (e.g., figure versus ground regions or the two regions in an ambiguous display). Modeling Offsets: An Example

As above, the model began by relaxing into its stable state for the first $50 \mathrm{~ms}$. Next, in addition to the Seg and Target inputs, an initial boost of activation was provided by the Task input (see Figure 4). This initial boost in activation generated a large peak of activation in PF, capturing the encoding of the task relevant stimulus locations. Because the targets were presented at the beginning of the trial for sufficient duration, this information built peaks in WM, with the peak at the 'figure' location (left) growing to a greater strength due to the stronger input at this location. Note that the initial boost of activation from Task was removed after $200 \mathrm{~ms}$, reflecting the completion of the encoding process.

The sustained activation of peaks in WM decreased activation in PF until it fell below threshold due to activation from the shared Inhib layer. Target input then began to diminish at the target locations at varying SOAs $(26,50,100$ and $150 \mathrm{~ms})$, starting $550 \mathrm{~ms}$ after the beginning of the trial. As the first target began to offset, the strength of the input at this location (left) began decreasing from its full strength over the course of $110 \mathrm{~ms}$. After the designated SOA, the second target input began to recede with the same time course.

In the absence of the Target input, peaks in WM began to decrease in activation, eventually de-stabilizing as noise caused local activation to fall below threshold $(<0)$. With the WM peaks no longer above threshold, PF was released from inhibition and was free to build a peak of activation in response to the continued Seg and Task inputs. Activation in PF then projects into the response layer to generate a 'left' or 'right' response. As can be seen in the figure, the peak pierced threshold in PF at the figure's location ("left" in Figure 4D). 
Consequently, activation at that location in the response field rose above threshold, generating a 'left' response and indicating that the 'figure' target offset first.

\section{Simulation Method}

Simulations were conducted in MATLAB 7.2 (Mathworks, Inc., http://www.mathworks.com). Each time step within the simulator was equated to $1.33 \mathrm{~ms}$. The conditions simulated were separated by task (onset or offset TOJs) and display type (figureground, ambiguous, separated). Within each task and display type, we conducted 500 trials at 4 different SOAs $(26,50,100,150 \mathrm{~ms})$. Each SOA occurred twice for each display condition to simulate figure-first and ground-first trials. Therefore, each batch of simulations included a total of 24,000 trials. We ran 5 batches of simulations to examine the robustness of the simulation results across repetitions of the entire design.

To parameterize the response field, we included a low resting level (-30) initially to suppress responding until after the figure-ground assignment was established. The remaining parameters were adjusted such that the field operated as a winner-take-all field (strong global inhibition, see $k_{r_{-} i n h i b}$ in Table 1), and received sufficient input from PF to generate responses (see $c_{r u}$ in Table 1). Note that the final parameters had slightly different resting levels for the onset versus offset events (see $h_{\text {on }}$ and $h_{\text {off }}$ in Table 1). Conceptually, this reflects our hypothesis that there is a greater need for selective responding in the offset condition (i.e., less excitatory resting level) given the difficulty of this task.

The remaining model parameters were taken from Johnson et al. (2009) and adjusted as needed to enable the model to make TOJs. Several changes were made to the PF parameters. In the model, all decisions pass through this layer; thus, the model parameters have to be precisely tuned relative to the strength of input and the strength of the suppression from WM. Thus, we 
decreased the resting level relative to Johnson et al. to make this layer more selective overall $\left(h_{o n}\right.$ and $h_{\text {off }}$ in Table 1). We also decreased excitation in PF $\left(c_{u u}\right)$ and increased the width of the inhibitory projection $\left(\sigma_{u v}\right)$. Relatedly, we made the target inputs weaker and a bit broader $\left(c_{t a r g}\right.$, $\sigma_{\text {targ }}$ ) so they would not dominate activation in the model, enabling PF to detect more subtle differences.

In addition to these changes, we decreased the resting levels $(h)$ of the inhibitory and WM layers. In the latter case, this brought the WM layer closer to threshold, enabling the system to detect offset by losing a WM peak. The final changes involved the noise sources in the model. We increased the strength of spatially correlated noise $\left(c_{\text {noise }}\right)$ and decreased the spatial spread of this noise $\left(\sigma_{\text {noise }}\right)$. We also added two noise sources: noise on the resting levels $\left(c_{h \_n o i s e}\right.$ see Table 2) and noise on the Target input ( cinput_noise $_{\text {) }}$. The former helped de-stabilize WM peaks in the offset detection conditions. The latter helped ensure that sometimes the model would make errors, even in conditions were onset / offset detection was relatively easy.

Results

Data from the simulations are plotted in Figure 5, with the left panels displaying the average performance of the simulations and the right panels displaying empirical data from Lester et al. (2009) for onset judgments and Hecht and Vecera (2011) for offset judgments. Visual comparison of the onset and offset data suggests a substantial overlap between the simulation and the empirical data. In both tasks, the figure-ground functions show approximately the same direction and amount of shift. Additionally, the model replicated the differences between slopes of the functions for all conditions, demonstrating its ability to capture the behavioral effects. 
To quantify the fit of the model to the behavioral data, we calculated the root mean squared error (RMSE) between the simulations and empirical data across experiments. For each batch of simulations, the RMSE was calculated for each condition and then averaged to determine the overall fit of the simulated to the behavioral data (see Table 3). Thus, we compared a total of 96 means (and an additional 6 means determined by curve fits, see below) between the model and the empirical data. The average RMSE across each of the five simulations was 0.0447 (range: $0.0423-0.0491$ ). The model provided an excellent fit to the behavioral data.

After determining the best fit of the model, the logistic curve for each simulation was calculated (see Figure 5). These logistic curves were then used to calculate two performance measures for TOJs. First, we calculated the point of subjective simultaneity (PSS; i.e., $50 \%$ 'ground first' responses), which is the primary measure of the prior entry and temporal extension effects within a TOJ paradigm. Second, we used the best-fitting curves to calculate the just noticeable difference (JND), which is half of the difference between the SOA values required to produce $75 \%$ and $25 \%$ 'ground first' responses. The JND provides an estimate of the slope of the function as it is monotonically related to the function. See Tables 4 and 5 for a summary of each run of the simulation's PSS and JND as well as the behavioral averages for these values.

The PSS for onsets was $15.9 \mathrm{~ms}$ for figure-ground trials, $-1.0 \mathrm{~ms}$ for separated region trials, and -3.3 ms for ambiguous trials (see Table 4). These values are a close approximation to the average behavioral data for all three trial types (10.1, -0.5 , and $-2.7 \mathrm{~ms}$, respectively). The positive PSS for figure-ground trials indicates that the point at which the stimuli would be perceived as simultaneous requires the ground target to lead, similar to the behavioral data. No 
shift was observed in the ambiguous and separated region trials, as evidenced by the near-zero PSSs. Therefore, the model obtained a prior entry effect for figure-ground trials.

Upon calculating the JNDs, the model yielded estimates of $62.3 \mathrm{~ms}$ for figure-ground trials, $48.2 \mathrm{~ms}$ for separated regions, and $73.1 \mathrm{~ms}$ for ambiguous displays. Again, the ordering of these data is similar to the findings in the behavioral data (see Table 4), although the absolute fit with the behavioral data is not as strong as the PSS results. Interestingly, the model was able to predict the shallower slope for the ambiguous trials, relative to both figure-ground and separated region trials.

Analysis of the PSS for offsets indicated a PSS of -10.6 ms for figure-ground trials, compared to $-10.1 \mathrm{~ms}$ in the behavioral data (see Table 5). In contrast, the separated region and ambiguous trials yielded a PSS of $-2.7 \mathrm{~ms}$ and $-1.1 \mathrm{~ms}$, respectively, mirroring the behavioral data (1.8 ms and -1.3 ms, respectively). The negative PSS for figure-ground trials suggests the model requires the figure target to lead the ground target in order to accurately order the offset events, as did the participants in the behavioral experiments (i.e., a temporal extension effect). Again, the near-zero PSS for separated regions and ambiguous trials replicates the effects observed in the data.

The JNDs also fell close to the behavioral data (see Table 5). Figure-ground trials (29.3 $\mathrm{ms})$, separated regions (29.8 ms), and ambiguous trials $(30.7 \mathrm{~ms})$ were identical. Likewise, the behavioral data had not differed between these display types $(36.4 \mathrm{~ms}, 42.6 \mathrm{~ms}$, and $39.6 \mathrm{~ms}$, respectively). This suggests that the slopes of the functions were equivalent, as was sensitivity to the offset events across display types, though there was a greater range and generally higher SOAs in the empirical results.

Denser SOA Sampling 
An additional set of simulations ( 5 batches) was conducted with a denser sampling of SOAs $(13,26,39,50,65,80,100,125$, and $150 \mathrm{~ms})$ to provide a closer estimate of the PSS and JND statistics. The best-fitting logistic curve fits are dependent upon how SOAs are sampled; adding data points increases the precision of the curve fit due to a decreased influence of potential outliers. Although the behavioral experiments require sparse sampling of SOA due to time and other constraints (e.g., fatigue), we can readily sample the model across many SOAs. Thus, we conducted a second set of simulations to examine whether denser SOA sampling would yield less variation in the estimates of the PSS and JND, which are both calculated on the bestfitting curve, resulting in better fits with the PSS and JND values obtained in the behavioral experiments.

Figure 6 illustrates the curve fits for these simulations, and Tables 6 and 7 contain the PSS and JND values for all runs of the simulations. For onsets, the average PSS was now 14.1 $\mathrm{ms}$ for figure-ground trials, $-0.5 \mathrm{~ms}$ for separated regions, and $-1.9 \mathrm{~ms}$ for ambiguous trials. These estimates are less variable among runs of the simulation, and they are closer estimates of the behavioral data. The JNDs for figure-ground $(44.7 \mathrm{~ms})$, separated regions $(47.2 \mathrm{~ms})$ and ambiguous $(70.0 \mathrm{~ms})$ trials show a similar trend: the estimates are more stable and better approximate the data.

The same reduction in variance of estimates was found in the case of offsets. The PSS values were now $-10.3 \mathrm{~ms},-0.4 \mathrm{~ms}$, and $-0.7 \mathrm{~ms}$ for figure-ground, separated regions, and ambiguous trials, respectively. These estimates are all within $2.5 \mathrm{~ms}$ of the behavioral data. Once again, the JNDs did not vary across trials; figure-ground $(29.8 \mathrm{~ms})$, separated regions $(31.7$ $\mathrm{ms})$ and ambiguous (30.1 ms) trials did not demonstrate differences in the slopes of the function. 


\section{Discussion}

In the present report, we demonstrated that a DNF model of change detection could be extended to explain the prior entry and temporal extension effects. To date, these effects have not been explained within a unified framework. Thus, the modeling work reported here makes a substantive contribution. Of course, common processing dynamics between change detection and temporal order judgments does not imply common neural mechanisms; the visual system could, for example, use the same processing dynamics in different regions for different tasks. Nevertheless, two very different tasks on the surface need not rely on radically different mechanisms.

Interestingly, the model not only captured the shifts in temporal perception as a result of figure-ground organization, but it also was able to produce the differences in sensitivity (i.e., slope differences) in the onset judgments while retaining the lack of differences in the offset judgments. Even more, the model accomplished this with relatively modest changes to the parameters used by Johnson and colleagues (Johnson, Spencer, \& Schöner, 2009; Johnson et al., 2009), even though the TOJ task, at face value, is quite different from the one-shot change detection task typically used in working memory experiments.

One critical component that underlies the model's TOJ performance is the structure of the segmentation input. When conducting initial tests of the parameters, the input coming from Seg impacted the amount of shift of the PSS. Specifically, shifts in the PSS only occurred when asymmetric input was provided to the model. The ambiguous and separated trials did not produce systematic shifts in the PSS. Therefore, one critical assumption of the DNF model presented here is that figure-ground organization is treated as an asymmetric input. 
For figure-ground trials, the PSS shifts away from zero due to increased errors in judgments at the shortest SOA - a consequence of the asymmetric activation projected from Seg. For onsets, this is the positive (ground-first) SOA, and for offsets it is the negative (figure-first) SOA. In the case of onsets shown in Figure 7, Seg's input is strong enough to keep the activation at the figure location slightly higher than the ground. As the first target input (i.e., at the ground location) is presented, activation at that location begins to rise. To make an accurate judgment, the ground's peak must overcome the difference in activation between the figure and ground before the figure target onsets. On a higher proportion of trials than when the figure's target leads, $26 \mathrm{~ms}$ is not a sufficient amount of time for the ground activation to recover, surpass the figure's activation, and begin projecting activation to the response field. Thus, an incorrect response is generated (i.e., 'figure') relative to the comparable figure-first SOA (26 ms).

Similarly, the shift in offset PSS is directly impacted by Seg's asymmetric projection. Again, raised levels of input for the figure location increases its peak activation in PF, which consequently builds a stronger peak in WM. Therefore, the 'disadvantaged' ground, in the case of offsets, is actually at an advantage by having less activation. As portrayed in Figure 8, less activation at the ground's location allows the peak in WM to fall below sub-threshold levels of activation (i.e., destabilize) sooner than the figure. At the shortest figure-first SOA (26 ms), the higher amount of activation at the figure's location is not always able to diminish rapidly enough to release inhibition in PF, which would allow a peak to be built in PF in response to the Task input. Instead, the ground peak destabilizes earlier in response to the offset of the corresponding target at its location, causing the incorrect response (i.e., 'ground') to be generated.

Clearly the asymmetric input from Seg played a critical role in producing the shift in the psychophysical functions. Critically, there is robust behavioral and neural support for this 
property of the model suggesting that neural activation differs between figures and grounds. For example, Vecera and O'Reilly generated and tested a model to explain the increased salience of foreground figures relative to ground regions (see Vecera \& O'Reilly, 1998, 2000; also see Kienker, Sejnowski, Hinton, \& Schumacher, 1986; Sejnowski \& Hinton, 1987; but see Peterson, 1999, and Peterson \& Skow, 2008 for an alternative account). Their interactive model contained neural representations of both figures and grounds. They predicted that figures have an enhanced neural activation compared to grounds. Vecera and O'Reilly's simulations provided strong support for their hypothesis, as opposed to other descriptive models that generated behavioral predictions that were tested in additional experiments (see Peterson, 1999, and Peterson \& Skow, 2008). Supporting Vecera and O’Reilly's conclusions, neurophysiological studies have demonstrated that perceiving figures results in increased neuronal firing relative to perceiving grounds. For example, V1 and V2 neurons in macaques increased in firing when their receptive fields were located on the figure (e.g., Lamme, 1995; Marcus \& Van Essen, 2002; Qiu, Sugihara, \& von der Heydt, 2007). Therefore, it is reasonable to assume that the Seg input is asymmetrical between figures and grounds and can be a critical factor in generating consequences of figure-ground organization (e.g., prior entry and temporal extension effects). In addition to impacting performance in the figure-ground conditions, the Seg input also impacted the model's performance in the ambiguous and separated regions conditions. The separated regions displays were assumed to lack segmentation input because these displays should be interpreted as two distinct objects. Thus, by not sharing a contour, there is no competition between the regions for figural assignment. In contrast, there is some competition in the figure-ground displays, but the majority of the competition can be resolved early in the trial, prior to the onset or offset events, and remains consistent throughout the trial. 
In the case of an ambiguous display, strong input was provided at both locations. We conjecture this reflected constant competition between these regions for figural assignment. The displays presented in the behavioral experiments contained an abrupt transition between regions along a straight, shared contour (see Figure 2; also see Figure 1 in Hecht \& Vecera, 2011). The regions appeared to accidentally align, which may have increased confusion in how to perceptually organize the display. The behavior of the model suggests that the increased input raises activation closer to threshold, making the system more susceptible to noise, which then could push either location's activation over threshold in PF (onsets) to generate a response, thereby reducing the accuracy of the TOJs.

Supporting this interpretation, Vecera et al. (2002) presented data in which ambiguous trials demonstrated a reaction time benefit over figure-ground displays. Observers viewed tworegion displays whose shared contour was aligned horizontally (figure-ground trials) or vertically (ambiguous trials). They demonstrated that the horizontal alignment (i.e., one region in the upper visual field and one in the lower) produced a reaction time benefit for lower regions (i.e., figure) over the upper regions in a memory matching task in which participants chose which of two regions was present in the previous two-region display. Interestingly, trials in which memory for ambiguous trials (regions to the left and right of each other) was probed yielded overall faster reaction times and higher accuracy than trials in which the regions appeared above and below each other. They concluded this benefit for ambiguous displays may result from fewer constraints placed on the ambiguous displays, allowing them to be processed more quickly than figure-ground displays (see also Driver \& Baylis, 1996). The current DNF model agrees with Vecera et al.'s (2002) discussion and suggests that fewer constraints may increase activation 
associated with processing these ambiguous regions, which can also speed response generation relative to figure-ground trials.

Interestingly, the strength of the Seg input did not impact the JND for ambiguous trials in the offset task: all conditions yielded the same slopes. This result highlights the difference between the onset and offset tasks. For onsets, the performance of the system is highly dependent upon, and sensitive to, the input. Slight variations in the input strength can shift the slope of the function. In contrast, offsets are not as strongly impacted by the strength of the input. Because offset judgments rely on the WM peaks falling back below threshold, and not in establishing the initial peaks in $\mathrm{PF}$, the strength of the input is not as critical in determining the slope of the function. Sensitivity to noise remains the same and differences between conditions instead rely on the interaction and inhibition between the two locations.

The strength of the input also interacted with the symmetry of input to influence the figure-ground PSS. Providing asymmetric input shifted the PSS, and the strength of the asymmetric input determined the size of the PSS: stronger inputs yielded larger shifts in the PSS (i.e., higher estimates of the SOA at 50\% 'ground first' responses). One prediction that can then be tested is to manipulate the strength of the input at either location, such as manipulating saliency of the figure. As the saliency of the figure increases, the PSS should continue to become larger, meaning that more time would be required between the figure and ground events to accurately order them.

The interaction between these two components (i.e., asymmetric input and strength of input) also offers an explanation for other aspects of the empirical data. Individual curve-fits from the prior entry and the temporal extension experiments (e.g., see supplementary files in Hecht \& Vecera, 2011) showed noticeable variation in both the PSS and the JND for both figure- 
ground and ambiguous trials upon visual inspection. Although a majority of the participants had the corresponding shifts in PSS for onset and offset tasks, other participants deviated from the patterns. One account for these individuals' performance is that their perception of the display is changed relative to those fitting the trends. Figure-ground organization, although often similar across individuals, is still a heuristic used by the visual system. The cues that are used to complete figure-ground organization (e.g., convexity) are not used consistently across individuals. Individuals whose PSS did not noticeably shift, for example, may have established a weaker representation of the convex region as figure, while those with opposite shifts likely established a reverse assignment (i.e., concave region as figure), on a greater proportion of trials. Similarly, the perception of the ambiguous trials can differ across participants, changing the strength of the input in accordance with changes in constraints implemented when processing the display. The DNF model gives us a tool to explore these individual differences and a framework within which one can test these specific proposals. One avenue for future exploration would be to measure figure-ground perception empirically across our various displays (figure-ground, ambiguous, and separated) and to use this to set the Seg input to the model, permitting us to more closely simulate performance across different display types. Such an approach would also lend itself to showing that the magnitude of TOJ effects is directly tied to the strength of figureground assignment. Specifically, displays that produced stronger figure-ground percepts should produce larger TOJ effects, and this could be established by measuring figure-ground perception for various displays and then using these displays in a TOJ task.

Individual differences might also result from sparse sampling of SOA. This methodological point is critical when considering individual differences in JND, though it also impacts the calculation of PSS. By obtaining a smaller sampling across SOA, fewer points are 
used to fit the psychophysical curves to each individual's data. As a result, the curve-fits are more likely to be impacted by outliers. Denser sampling across SOA in the model supports this conjecture: the five batches of denser sampling simulations provided closer estimates of the PSS and JND to one another than did the five batches using the SOAs tested empirically (see Table 3 and compare Tables 4 to 6 and Tables 5 to 7). Therefore, the individual data likely include the impact of differences in curve-fits on the JND and PSS; denser sampling may better estimate each individual's psychophysical function and reduce variation between individuals. Of course, due to constraints (e.g., time) this hypothesis may only be tested using psychophysically trained participants, though other concerns (e.g., fatigue) remain.

Beyond accounting for figure-ground phenomena, our model can be extended to the attention literature. Other manipulations that produce asymmetric input, such as spatial attention, may also be provided to the model, in lieu of Seg, to the change detection mechanism to produce these same consequences for temporal processing. Figure-ground organization and attention do affect temporal processing in similar ways: both processes result in prior entry and temporal extension effects (see Lester et al., 2009, and Hecht \& Vecera, 2011, for a discussion). Therefore, we surmise that these processes may utilize the same mechanism in order to influence temporal processing in the same, structured fashion. By extension, current theories regarding spatial attention's impact on temporal processing may provide further insight into the nature of the mechanism implemented in the DNF model. The current model suggests that raised activation (i.e., asymmetric input) for figures relative to grounds puts activation closer to detection threshold in the case of onsets, but can also extend the duration of processing for these regions as well. These processing dynamics also apply to recent evidence that indicates a 'prior entry’ effect for convex shapes and near surfaces (West, Pratt, \& Peterson, 2013), similar to that 
observed for figural regions. Such results suggest that surface convexity and monocular depth might produce asymmetric inputs similar to those from figure-ground perception, and the DNF model would account for these results with the mechanisms we have outlined here.

Similar accounts have been proposed within the attention literature, but they rely on separate mechanisms: one to account for prior entry and one to account for temporal extension. Schneider and Bavelier (2003) found that the prior entry effect can be attributed to both an acceleration of processing and to sensory facilitation, whereby perceptual processing is accelerated (e.g., neurally enhanced) independently of attention. Their proposal suggested that neurons responding to attended regions increased in activation, enhancing their representation. This enhancement then allowed for faster detection of targets appearing at that location. This general account aligns with the DNF model, which also uses an increase in activation in response to the source of the asymmetric, or biased, input (i.e., figure-ground organization).

An alternative theory was proposed to account for the temporal extension effect. In particular, Yeshurun and Levy (2003) considered two alternatives: spatial and temporal resolution may be independent, separable processes (e.g., Lehky, 1985; Wilson, 1980) or that they interact (e.g., Carrasco, 1990; Drum, 1984), yielding a tradeoff between the two (e.g., Wilson, 1980; Wilson \& Bergen, 1979) where foveated locations are provided higher spatial resolution and the periphery is provided higher temporal resolution. Using a gap detection task to assess spatial resolution and a two-flicker fusion threshold paradigm (e.g., Levine, 2000) to assess temporal resolution, Yeshurun and Levy found behavioral support for a tradeoff between the two processes.

After finding that attended locations had high spatial and low temporal resolution, Yeshurun and Levy (2003) outlined a neurophysiological hypothesis that accounted for these 
behavioral effects. This hypothesis considered the processing differences between foveated and peripheral locations, and drew parallels between these differences and the characteristics associated with the parvocellular (P) and magnocellular (M) pathways in visual processing. Parvocellular neurons have smaller receptive fields (e.g., Schiller \& Logothetis, 1990; Shapley \& Perry, 1986) and extended response durations (e.g., Merigan \& Maunsell, 1993; Schiller \& Logothetis, 1990), contrasting with the properties of M neurons. Moreover, these characteristics align with the differences found between foveated and peripheral locations (e.g., Allen \& Hess, 1992). Therefore, enhancing one domain (spatial or temporal resolution) should result in a decrease to the other domain, creating a tradeoff between the two. By Yeshurun and Levy's account, attention increases the activation of $\mathrm{P}$ neurons. In turn, this activation then projects inhibition to the $\mathrm{M}$ neurons resulting in higher spatial acuity but poorer temporal resolution at the location of attention.

Yeshurun (2004) later supported this neurophysiological hypothesis in a series of behavioral experiments examining temporal resolution using a two-flicker fusion threshold paradigm. Critically, these studies were designed to inhibit the M neurons prior to activation of the $\mathrm{P}$ neurons. In doing so, the $\mathrm{P}$ neurons, when activated via attention, would not be able to inhibit the M neurons. Yeshurun inhibited the M pathway by presenting either colored stimuli in isoluminant pairs (e.g., blue paired with yellow) or a diffuse red light. Magnocellular neurons are both colorblind (e.g., Merigan \& Maunsell, 1993; Schiller \& Logothetis, 1990) and are suppressed by diffuse red light falling on the surround of their receptive fields (e.g., Livingstone \& Hubel, 1984; Van Essen, 1985). If the inhibition of M by P neurons while processing information at an attended location is critical in degrading temporal resolution, then, Yeshurun argued, eliminating this inhibitory process should improve temporal resolution at attended 
regions. Yeshurun (2004) found decrements in temporal resolution under normal circumstances, but found attenuated decrements when the flicker stimuli were isoluminant and found no differences in resolution between attended and unattended locations when a diffuse red background was used. These results supported the neurophysiological hypothesis that P-M inhibition processes impact temporal discrimination by decreasing temporal resolution.

Furthermore, Yeshurun's results offer an explanation for the observation that $\mathrm{P}$ neurons are active for longer durations and have a slower rate of decay relative to $\mathrm{M}$ neurons (e.g., Merigan \& Maunsell, 1993; Schiller \& Logothetis, 1990).

Therefore, Yeshurun and Levy's (2003; see also Yeshurun, 2004) neurophysiological hypothesis addresses the physical mechanism that can give rise to the temporal extension effect (i.e., extended processing for attended locations or figures). Figure-ground organization has a similar impact on temporal resolution, such that figures are processed similarly to attended locations. Specifically, the temporal resolution is degraded at these locations (Hecht \& Vecera, 2011, 2014). Yeshurun and Levy's account is reminiscent of Weisstein, Maguire, and Brannan's (1992) proposal that regions processed by the P pathway are recognized as figures, suggesting that attention and figure-ground may be utilizing similar mechanisms. Because figure-ground has been shown to impact temporal processing in similar ways as attention, potentially resulting in similar neurophysiological patterns, we speculate that temporal processing decrements from figure-ground organization and from attention are generated from use of the same mechanism, as demonstrated by the DNF model, and that the source of this mechanism may relate to the P and M pathways.

Still, it is important to note that there is no direct evidence for the P-M inhibition hypothesis. Indirect behavioral evidence has been provided (e.g., Yeshurun, 2004) to support the 
hypothesis, but currently no studies have examined activation patterns in these pathways during completion of TOJ tasks to study the role and interaction of the $\mathrm{P}$ and M pathways. As Schiller (1996; see also Schiller \& Logothetis, 1990) noted, the separation of these pathways is less distinct in later visual processing (e.g., V4). Without directly studying the neurophysiological functioning in these tasks, it is uncertain whether theories should distinguish between the P and M pathways. Note that a recent DNF model by Schneegans et al. (2014) further integrates perception, attention, and visual working memory, showing how the contents of visual working memory affect perception which, in turn, influences the allocation of attention and saccadic eye movements (see Hollingworth et al., 2013). Because the Schneegans et al. (2014) model has separate pathways for spatial attention and a surface feature pathway, it might serve to bridge our current DNF model to Yeshurun's (2004) two-pathway account of temporal processing. Regardless, the current DNF model provides a detailed depiction of the neural dynamics (i.e., patterns of activation and their interaction) that can produce the prior entry and temporal extension effects in figure-ground perception and in the allocation of spatial attention. 


\section{References}

Allen, D., \& Hess, R. F. (1992). Is the visual field temporally homogeneous? Vision Research, 32, 1075-1084.

Amari, S. (1977). Dynamics of pattern formation in lateral-inhibition type neural fields. Biological Cybernetics, 27, 77-87.

Baylis, G. C., \& Driver, J. (1995). Obligatory edge assignment in vision: The role of figure and part segmentation in symmetry detection. Journal of Experiment Psychology: Human Perception \& Performance, 21, 1323-1342.

Carrasco, M. (1990). Visual space-time interactions: Effects of adapting to spatial frequencies on temporal sensitivity. Perception \& Psychophysics, 48, 488-496.

Douglas, R., \& Martin, K. (1998). Neocortex. In G. M. Shepherd (Ed.), The Synaptic Organization of the Brain (4th ed., pp. 459-509). New York: Oxford University Press.

Driver, J., \& Baylis, G. C. (1996). Edge-assignment and figure-ground segmentation in shortterm visual matching. Cognitive Psychology, 31, 248-306.

Hecht, L. N., \& Vecera, S. P. (2011). Delayed offset detection on figures relative to backgrounds. Journal of Vision, 11(13), 1-8. doi:10.1167/11.13.15

Hecht, L. N., \& Vecera, S. P. (2014). Temporal resolution of figures and grounds. Acta Psychologica, 147, 147-151. doi:10.1016/j.actpsy.2013.06.010

Hein, E., Rolke, B., \& Ulrich, R. (2006). Visual attention and temporal discrimination: Differential effects of automatic and voluntary cueing. Visual Cognition, 13, 29-50.

Johnson, J. S., Spencer, J. P., Luck, S. J., \& Schöner, G. (2009). A dynamic neural field model of visual working memory and change detection. Psychological Science, 20, 568-577. 
Johnson, J. S., Spencer, J. P., \& Schöner, G. (2009). A layered neural architecture for the consolidation, maintenance, and updating of representations in visual working memory. Brain Research, 1299, 17-32.

Julesz, B. (1984). A brief outline of the texton theory of human vision. Trends in Neurosciences, $6,41-45$.

Kienker, P. K., Sejnowski, T. J., Hinton, G. E., \& Schumacher, L. E. (1986). Separating figure from ground with a parallel network. Perception, 15, 197-216.

Klymenko, V., \& Weisstein, N. (1986). Spatial frequency differences can determine figureground organization. Journal of Experimental Psychology: Human Perception and Performance, 12, 324-330.

Klymenko, V., \& Weisstein, N. (1989a). Figure and ground in space and time: 1. Temporal response surfaces of perceptual organization. Perception, 18, 627-637.

Klymenko, V., \& Weisstein, N. (1989b). Figure and ground in space and time: 2. Frequency, velocity, and perceptual organization. Perception, 18, 639-648.

Klymenko, V., Weisstein, N., Topolski, R., \& Hsieh, C-H. (1989). Spatial and temporal frequency in figure-ground organization. Perception \& Psychophysics, 45, 395-403.

Lamme, V. A. F. (1995). The neurophysiology of figure-ground segregation in primary visual cortex. Journal of Neuroscience, 15, 1605-1615.

Lazareva, O. F., Castro, L., Vecera, S. P., \& Wasserman, E. A. (2006). Figure-ground assignment in pigeons: Evidence for a figural benefit. Perception \& Psychophysics, $68(5), 711-724$

Lehky, S. R. (1985). Temporal properties of visual channels measured by masking. Journal of the Optical Society of America, 2, 1260-1272. 
Lester, B. D., Hecht, L. N., \& Vecera, S. P. (2009). Visual prior entry for foreground figures. Psychonomic Bulletin \& Review.

Levine, M. W. (2000). Fundamentals of sensation and perception. New York: Oxford University Press.

Livingstone, M., \& Hubel, D. H. (1984). Anatomy and physiology of a color system in the primate visual cortex. Journal of Neuroscience, 4, 309-356.

Luck, S. J., \& Vogel, E. K. (1997). The capacity of visual working memory for features and conjunctions. Nature, 390, 279-281.

Marcus, D. S., \& Van Essen, D. C. 2002). Scene segmentation and attention in primate cortical areas V1 and V2. Journal of Neurophysiology, 88, 2648-2658.

Merigan, W. H., \& Maunsell, J. H. R. (1993). How parallel are the primate visual pathways? Annual Review of Neuroscience, 16, 369-402.

Nelson, R. A., \& Palmer, S. E. (2007). Familiar shapes attract attention in figure-ground displays. Perception \& Psychophysics, 69(3), 382-392.

Palmer, S. E. (1999). Vision science: Photons to phenomenology. Cambridge, Massachusetts: MIT Press.

Palmer, S. E. (2002). Perceptual organization in vision. In H. Pashler \& S. Yantis (Eds.), Stevens' handbook of experimental psychology: Volume 1. Sensation and perception (pp. 177-234). New York: Wiley.

Palmer, S., \& Rock, I. (1994). Rethinking perceptual organization: The role of uniform connectedness. Psychonomic Bulletin \& Review, 1, 29-55.

Peterson, M. A. (1994). Object recognition processes can and do operate before figure-ground organization. Current Directions in Psychological Science, 3, 105-111. 
Peterson, M. A. (1999). What's in a stage name? Comment on Vecera and O'Reilly (1998). Journal of Experimental Psychology: Human Perception and Performance, 25, 276-286.

Peterson, M. A., \& Gibson, B. S. (1991). The initial identification of figure-ground relationships: Contributions from shape recognition routines. Bulletin of the Psychonomic Society, 29, 199-202.

Peterson, M. A., \& Gibson, B. S. (1993). Shape recognition contributions to figure-ground organization in three-dimensional displays. Cognitive Psychology, 25, 383-429.

Peterson, M. A., \& Gibson, B. S. (1994). Must figure-ground organization precede object recognition? An assumption in peril. Psychological Science, 5, 253-259.

Peterson, M. A., Harvey, E. H., \& Weidenbacher, H. L. (1991). Shape recognition inputs to figure-ground organization: Which route counts? Journal of Experimental Psychology: Human Perception and Performance, 17, 1075-1089.

Peterson, M. A., \& Skow, E. (2008). Inhibitory competition between shape properties in figureground perception. Journal of Experimental Psychology: Human Perception and Performance, 34, 251-267.

Pomerantz, J. R., \& Kubovy, M. (1986). Theoretical approaches to perceptual organization: Simplicity and likelihood principles. In K. R. Boff, L. Kaufman \& J. P. Thomas (Eds.), Handbook of perception and human performance: Vol. 2 Cognitive processes and performance (pp. 1-46). New York: Wiley.

Qiu, F.T., Sugihara, T., von der Heydt, R. (2007). Figure-ground mechanisms provide structure for selective attention. Nature Neuroscience, 10, 1492-1499.

Rock, I. (1975). An introduction to perception. New York: Macmillan.

Rock, I. (1995). Perception. New York: Scientific American Books. 
Roelfsema, P. R., Lamme, V. A., Spekreijse, H., \& Bosch, H. (2002). Figure-ground segregation in a recurrent network architecture. Journal of Cognitive Neuroscience, 14(4), 525-537.

Rolke, B., Ulrich, R., \& Bausenhart, K. M. (2006). Attention delays perceived stimulus offset. Vision Research, 46, 2926-2933.

Rubin, E. (1915/1958). Figure and ground. In D. C. Beardslee \& M. Wertheimer (Eds.), Readings in perception (pp. 194-203). Princeton, NJ: Van Nostrand.

Schiller, P. H. (1996). On the specificity of neurons and visual areas. Behavioural Brain Research, 76, 21-35.

Schiller, P. H., \& Logothetis, N. K. (1990). The color-opponent and broad-band channels in the primate visual system. Trends in Neuroscience, 13, 392-398.

Schneider, K. A., \& Bavelier, D. (2003). Components of visual prior entry. Cognitive Psychology, 47, 333-366.

Schneegans, S., Spencer, J. P., Schöner, G., Hwang, S., \& Hollingworth, A. (2014). Dynamic interactions between visual working memory and saccade target selection. Journal of vision, 14(11), 9 .

Sejnowski, T. J., \& Hinton, G. E. (1987). Separating figure from ground with a boltzmann machine. In M. A. Arbib \& A. R. Hanson (Eds.), Vision, brain, and cooperative computation (pp. 703-724). Cambridge, MA: MIT.

Self, M. W., Mookhoek, A., Tjalma, N., \& Roelfsema, P. R. (2015). Contextual effects on perceived contrast: Figure-ground assignment and orientation contrast. Journal of Vision, 15(2), 2.

Shapley, R., \& Perry, V. H. (1986). Cat and monkey retinal ganglion cells and their visual functional roles. Trends in Neuroscience, 9, 229-235. 
Shore, D. I., \& Spence, C. (2005). Prior entry. In L. Itti \& G. Rees \& J. K. Tsostos (Eds.), Neurobiology of attention (pp. 89-95). New York: Elsevier.

Shore, D. I., Spence, C., \& Klein, R. K. (2001). Visual prior entry. Psychological Science, 12, 205-212.

Simmering, V. R., Schutte, A. R., \& Spencer, J. P. (2007). Generalizing the dynamic field theory of spatial cognition across real and developmental time scales. Brain Research, 1202, 6886.

Spencer, J. P., Perone, S., \& Johnson, J. S. (2009). The dynamic field theory and embodied cognitive dynamics. In J.P. Spencer, M.S. Thomas, \& J.L. McClelland (Eds.) Toward a unified theory of cevelopment: Connectionism and dynamic systems theory re-considered (p. 86-118). New York: Oxford University Press.

Spencer, J. P., Simmering, V. R., Schutte, A. R., \& Schöner, G. (2007). What does theoretical neuroscience have to offer the study of behavioral development? Insights from a dynamic field theory of spatial cognition. In J. Plumert \& J. P. Spencer (Eds.) The emerging spatial mind (p. 320-361). New York: Oxford University Press.

Van Essen, D. C. (1985). Functional organization of primate visual cortex. In E. G. Jones \& A. A. Peters (Eds.), Cerebral cortex (vol. 3, pp. 259-329). New York: Plenum Press.

Vecera, S. P., Flevaris, A. V., \& Filapek, J. C. (2004). Exogenous spatial attention influences figure-ground organization. Psychological Science, 15, 20-26.

Vecera, S. P., \& O’Reilly, R. C. (1998). Figure-ground organization and object recognition processes: An interactive account. Journal of Experimental Psychology: Human Perception and Performance, 24, 441-462. 
Vecera, S. P., \& O’Reilly, R. C. (2000). Graded effects in hierarchical figure-ground organization: A reply to Peterson (1999). Journal of Experimental Psychology: Human Perception and Performance, 26, 1221-1231.

Vecera, S. P., Vogel, E. K., \& Woodman, G. F. (2002). Lower region: A new cue for figureground organization. Journal of Experimental Psychology: General, 131, 194-205.

Weisstein, N., Maguire, W., \& Brannan, J. R. (1992). M and P pathways and the perception of figure and ground. In J. R. Brannan (Ed.), Applications of parallel processing in vision. New York: Elsevier.

Wilson, H. R. (1980). Spatiotemporal characterization of a transient mechanism in the human visual system. Vision Research, 20, 443-452.

Wilson, H. R., \& Bergen, J. R. (1979). A four mechanism model for threshold and spatial vision. Vision Research, 19, 19-32.

Wilson, H. R., \& Cowan, J. D. (1972). Excitatory and inhibitory interactions in localized populations of model neurons. Biophysical Journal, 12, 1-24.

Wong, E., \& Weisstein, N. (1982). A new perceptual context-superiority effect: Line segments are more visible against a figure than against a ground. Science, 218(4572), 587-589.

Wong, E., \& Weisstein, N. (1983). Sharp targets are detected better against a figure, and blurred targets are detected better against a background. Journal of Experimental Psychology: Human Perception and Performance, 9(2), 194-202.

Yeshurun, Y. (2004). Isoluminant stimuli and red background attenuate the effects of transient spatial attention on temporal resolution. Vision Research, 44, 1375-1387.

Yeshurun, Y., \& Levy, L. (2003). Transient spatial attention degrades temporal resolution. Psychological Science, 14, 225-231. 
Table 1. Parameter Values Used in Model Simulations

\begin{tabular}{|c|c|c|c|c|c|c|c|c|}
\hline Layer & $h_{\text {init }}$ & $h_{o n}$ & $h_{o f f}$ & $\begin{array}{l}\text { Self- } \\
\text { excitation }\end{array}$ & $\begin{array}{l}\text { Excitatory } \\
\text { projections }\end{array}$ & $\begin{array}{l}\text { Inhibitory } \\
\text { projections }\end{array}$ & $\begin{array}{l}\text { Scaled } \\
\text { Input } \\
\text { Strength }\end{array}$ & $\tau$ \\
\hline \multirow[t]{2}{*}{$u(\mathrm{PF})$} & & $\begin{array}{l}-19 \\
(-7)\end{array}$ & -14 & $\begin{aligned} \mathrm{c}_{u u}= & 3.15 \\
& (2.0)\end{aligned}$ & $c_{u r}=1.0^{*}$ & $c_{u v}=1.85$ & $I_{u}=1.0$ & 80 \\
\hline & & & & $\sigma_{u u}=3$ & $\sigma_{u r}=5^{*}$ & $\begin{aligned} \sigma_{u v}= & 24 \\
& (26) \\
k_{u v}= & .05\end{aligned}$ & & \\
\hline \multirow[t]{3}{*}{$v($ Inhib) } & & $\begin{array}{l}-8.5 \\
(-12)\end{array}$ & -8.5 & & $c_{v u}=2.0$ & & & 10 \\
\hline & & & & & $\sigma_{v u}=10$ & & & \\
\hline & & & & & $\begin{array}{l}c_{v w}=1.95 \\
\sigma_{v w}=5\end{array}$ & & & \\
\hline \multirow[t]{2}{*}{$w(\mathrm{WM})$} & & $\begin{array}{l}-13 \\
(-4)\end{array}$ & -13 & $c_{w w}=3.15$ & $c_{w u}=1.5$ & $c_{w v}=.325$ & $I_{w}=0.2$ & 80 \\
\hline & & & & $\sigma_{w w}=3$ & $\sigma_{w u}=5$ & $\begin{array}{l}\sigma_{w v}=42 \\
k_{w v}=.08\end{array}$ & & \\
\hline$r(\mathrm{RESP})^{*}$ & -30 & -7 & -8 & $\begin{array}{l}\mathrm{c}_{r_{-} \text {excite }}=5 \\
\sigma_{r_{-} \text {excite }}=5\end{array}$ & $\begin{aligned} c_{r u} & =1.0 \\
\sigma_{r u} & =5\end{aligned}$ & $k_{r_{-} i n h i b}=3$ & $I_{r}=0.2$ & 80 \\
\hline
\end{tabular}

* all parameters associated with the RESP field were new relative to Johnson, Spencer, Luck, et al. (2009). Other parameters that differed relative to Johnson et al. are indicated in parentheses. 
Table 2. Additional Parameter Values Used in Model Simulations

Strength of spatially correlated noise to fields $c_{\text {noise }}=.1(.04)$

Width of spatially correlated noise

$\sigma_{\text {noise }}=0.45(1.0)$

Strength of noise on resting level $(h)$

$c_{h \_ \text {noise }}=3^{*}$

Strength of noise on Target input

$c_{\text {input } \text { noise }}=0.6^{*}$

Slope of sigmoid

$\beta=5.0$

Field size

$n=151$

Segmentation Inputs

$c_{\text {seg }}=0.6^{*}$

$\sigma_{s e g}=5^{*}$

$c_{\text {seg_amb }}=3 *$

$\sigma_{\text {seg_amb }}=5^{*}$

Target Input

$c_{\text {targ }}=20$ (30)

$\sigma_{\text {targ }}=5$ (3)

Task Input

$c_{\text {task }}=15^{*}$

$\sigma_{\text {task }}=5^{*}$

* Parameters that were new relative to Johnson, Spencer, Luck, et al. (2009). Other parameters that differed relative to Johnson et al. are indicated in parentheses. 
Table 3. Root Mean Squared Errors for All Simulations

\section{Simulations}

\begin{tabular}{lcccccc} 
Simulation & Run 1 & Run 2 & Run 3 & Run 4 & Run 5 & Average \\
\hline $\begin{array}{l}\text { SOAs Match Empirical } \\
\text { Studies }\end{array}$ & 0.0491 & 0.0423 & 0.0444 & 0.0446 & 0.0430 & 0.0447 \\
& & & & & & $(0.0027)$ \\
Denser Sampling of SOAs & 0.0457 & 0.0459 & 0.0430 & 0.0409 & 0.0403 & 0.0432 \\
& & & & & & $(0.0026)$ \\
\hline
\end{tabular}

Note: Parentheses indicate standard deviations. 
Table 4. Statistics for Onset Simulations

Simulations

\begin{tabular}{llccccccc} 
Statistic & Trial Type & $\begin{array}{c}\text { Behavioral } \\
\text { Data }\end{array}$ & $\begin{array}{c}\text { Run } \\
1\end{array}$ & $\begin{array}{c}\text { Run } \\
2\end{array}$ & $\begin{array}{c}\text { Run } \\
3\end{array}$ & $\begin{array}{c}\text { Run } \\
4\end{array}$ & $\begin{array}{c}\text { Run } \\
5\end{array}$ & Average \\
\hline PSS & Figure-ground & 10.1 & 17.9 & 14.5 & 13.2 & 16.4 & 17.7 & $\begin{array}{c}15.9 \\
(2.0)\end{array}$ \\
& & & & & & & & -1.0 \\
& Separated & -0.5 & -4.2 & -2.1 & 0.6 & 1.9 & -1.5 & $(2.4)$ \\
& Regions & & & & & & & -3.3 \\
& Ambiguous & -2.7 & -0.1 & -7.6 & -1.4 & -4.4 & -3.3 & $(2.9)$ \\
& & & & & & & \\
& & & & & & & 62.3 \\
JND & Figure-ground & 41.6 & 66.5 & 58.9 & 58.8 & 62.9 & 64.1 & $(3.3)$ \\
& & & & & & & 48.2 \\
& Separated & 39.8 & 52.5 & 47.4 & 46.6 & 46.3 & 48.2 & \\
& Regions & & & & & & & $(2.5)$ \\
& Ambiguous & 82.0 & 76.9 & 76.8 & 70.6 & 71.5 & 69.6 & 73.1 \\
& & & & & & & $(3.5)$ \\
\hline
\end{tabular}

Note: Parentheses indicate standard deviations. 
Table 5. Statistics for Offset Simulations

Simulations

\begin{tabular}{llccccccc} 
Statistic & Trial Type & $\begin{array}{c}\text { Behavioral } \\
\text { Data }\end{array}$ & $\begin{array}{c}\text { Run } \\
1\end{array}$ & $\begin{array}{c}\text { Run } \\
2\end{array}$ & $\begin{array}{c}\text { Run } \\
3\end{array}$ & $\begin{array}{c}\text { Run } \\
4\end{array}$ & $\begin{array}{c}\text { Run } \\
5\end{array}$ & Average \\
\hline PSS & $\begin{array}{l}\text { Figure- } \\
\text { ground }\end{array}$ & -10.1 & -12.4 & -9.5 & -10.0 & -10.5 & -10.3 & -10.6 \\
& $\begin{array}{l}\text { Separated } \\
\text { Regions }\end{array}$ & 1.8 & -5.0 & -4.3 & -2.2 & -1.0 & -1.2 & -2.7 \\
& & & & & & & & $(1.8)$ \\
& Ambiguous & -1.3 & -3.0 & -1.1 & 2.1 & -0.5 & -3.0 & -1.1 \\
& & & & & & & & $(2.1)$ \\
JND & Figure- & 36.4 & 30.1 & 30.5 & 29.4 & 27.9 & 28.5 & $\begin{array}{l}29.3 \\
\text { ground }\end{array}$ \\
& $\begin{array}{l}\text { Separated } \\
\text { Regions }\end{array}$ & 42.6 & 29.9 & 29.9 & 30.3 & 28.4 & 30.6 & $\begin{array}{l}29.8 \\
(1.1)\end{array}$ \\
& Ambiguous & 39.6 & 30.3 & 31.2 & 29.0 & 31.8 & 31.2 & 30.7 \\
& & & & & & & & $(1.1)$ \\
\hline
\end{tabular}

Note: Parentheses indicate standard deviations. 
Table 6. Statistics for Onset Simulations with Denser SOA Sampling

\section{Simulations}

\begin{tabular}{llccccccc} 
Statistic & Trial Type & $\begin{array}{c}\text { Behavioral } \\
\text { Data }\end{array}$ & $\begin{array}{c}\text { Run } \\
1\end{array}$ & $\begin{array}{c}\text { Run } \\
2\end{array}$ & $\begin{array}{c}\text { Run } \\
3\end{array}$ & $\begin{array}{c}\text { Run } \\
4\end{array}$ & $\begin{array}{c}\text { Run } \\
5\end{array}$ & Average \\
\hline PSS & $\begin{array}{l}\text { Figure- } \\
\text { ground }\end{array}$ & 10.1 & 15.7 & 11.8 & 14.5 & 13.1 & 15.1 & 14.1 \\
& $\begin{array}{l}\text { Separated } \\
\text { Regions }\end{array}$ & -0.5 & -0.7 & 0.3 & -1.6 & -0.6 & -0.1 & 0.5 \\
& Ambiguous & -2.7 & -2.1 & -3.2 & -2.9 & 0.9 & -2.3 & 1.9 \\
& & & & & & & & $(1.7)$ \\
& & & & & & & & \\
JND & $\begin{array}{l}\text { Figure- } \\
\text { ground }\end{array}$ & 41.6 & 43.7 & 45.9 & 56.6 & 43.2 & 45.0 & 44.7 \\
& $\begin{array}{l}\text { Separated } \\
\text { Regions }\end{array}$ & 39.8 & 48.3 & 47.5 & 46.5 & 45.5 & 48.0 & 47.2 \\
& & & & & & & & $(1.2)$ \\
& Ambiguous & 82.0 & 72.2 & 65.9 & 68.8 & 73.5 & 69.5 & 70.0 \\
& & & & & & & $(3.0)$ \\
\hline
\end{tabular}

Note: Parentheses indicate standard deviations. 
Table 7. Statistics for Offset Simulations with Denser SOA Sampling

Simulations

\begin{tabular}{llccccccc} 
Statistic & Trial Type & $\begin{array}{c}\text { Behavioral } \\
\text { Data }\end{array}$ & $\begin{array}{c}\text { Run } \\
1\end{array}$ & $\begin{array}{c}\text { Run } \\
2\end{array}$ & $\begin{array}{c}\text { Run } \\
3\end{array}$ & $\begin{array}{c}\text { Run } \\
4\end{array}$ & $\begin{array}{c}\text { Run } \\
5\end{array}$ & Average \\
\hline PSS & Figure-ground & -10.1 & -9.1 & -10.7 & -10.1 & -9.3 & -12.3 & -10.3 \\
& & & & & & & & $(1.3)$ \\
& Separated & 1.8 & 0.0 & 1.5 & 1.0 & 1.2 & 1.6 & 0.4 \\
& Regions & & & & & & & $(1.2)$ \\
& Ambiguous & -1.3 & -1.0 & -1.1 & -1.1 & 1.4 & -1.4 & 0.7 \\
& & & & & & & & $(1.1)$ \\
& & & & & & & \\
JND & Figure-ground & 36.4 & 29.1 & 28.1 & 31.2 & 29.8 & 30.7 & 29.8 \\
& & & & & & & & $(1.3)$ \\
& Separated & 42.6 & 30.1 & 32.7 & 30.1 & 32.4 & 32.8 & 31.7 \\
& Regions & & & & & & & $(1.4)$ \\
& Ambiguous & 39.6 & 30.9 & 29.7 & 29.6 & 30.4 & 29.9 & 30.1 \\
& & & & & & & & $(0.5)$ \\
\hline
\end{tabular}

Note: Parentheses indicate standard deviations. 


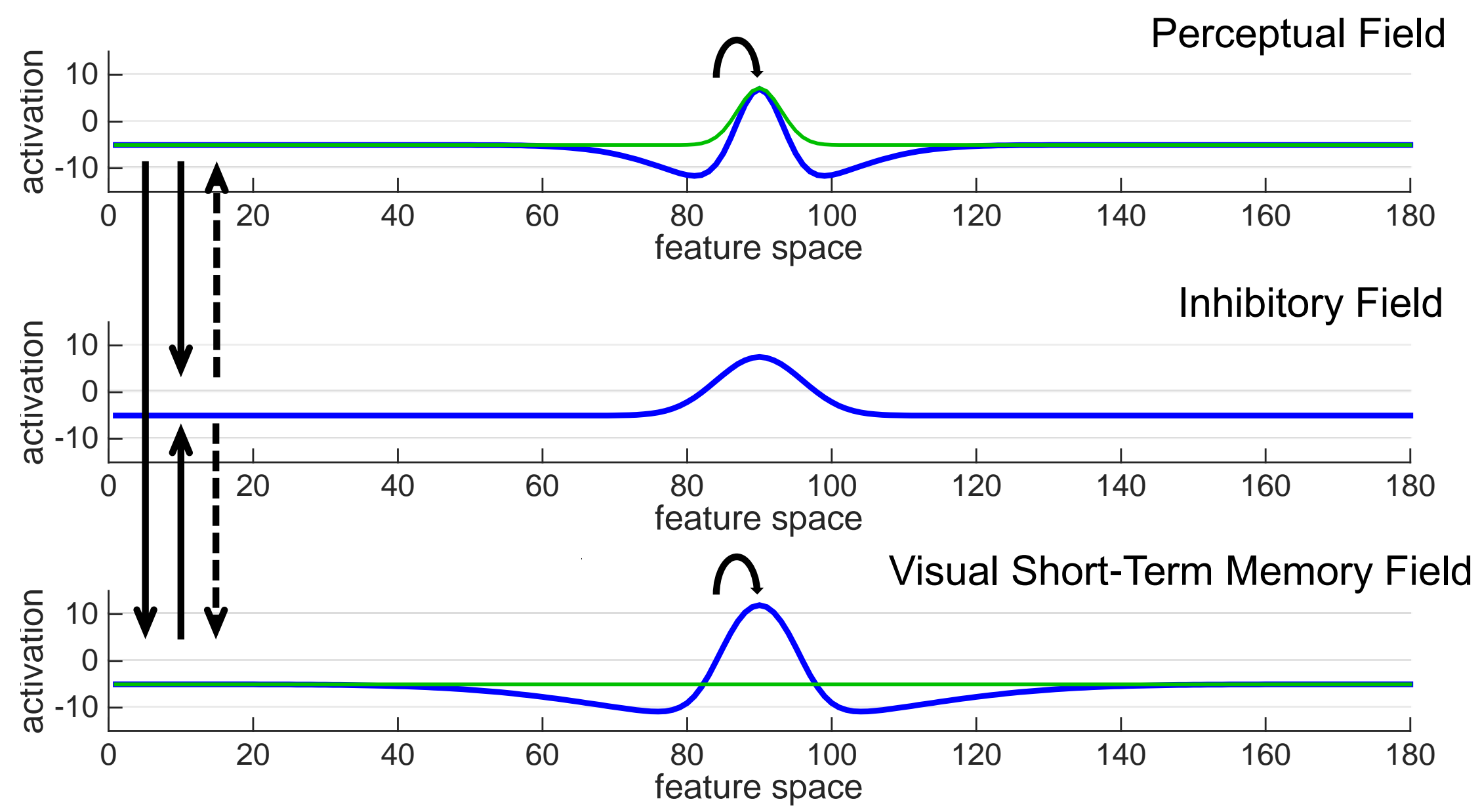

Figure 1. Basic structure of a three-layer DNF model. Solid arrows indicate excitation and dashed arrows indicate inhibition. All neurons are ordered according to functional topography based on a particular feature dimension (e.g., spatial location). For further details, see Johnson, Spencer, Luck, et al., 2009, or Johnson, Spencer, and Schöner, 2009. 
Figure 2. Examples of stimuli.

Figure-ground

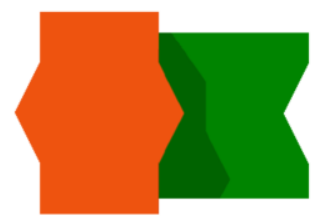

Figure-ground: Separated

Ambiguous:

Convex
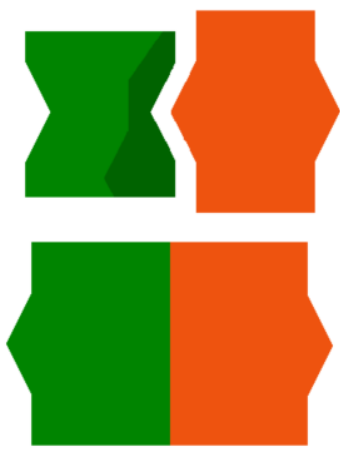

Ambiguous:

Concave

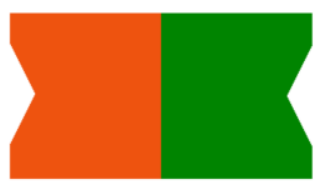


$\underline{\text { Time }}$

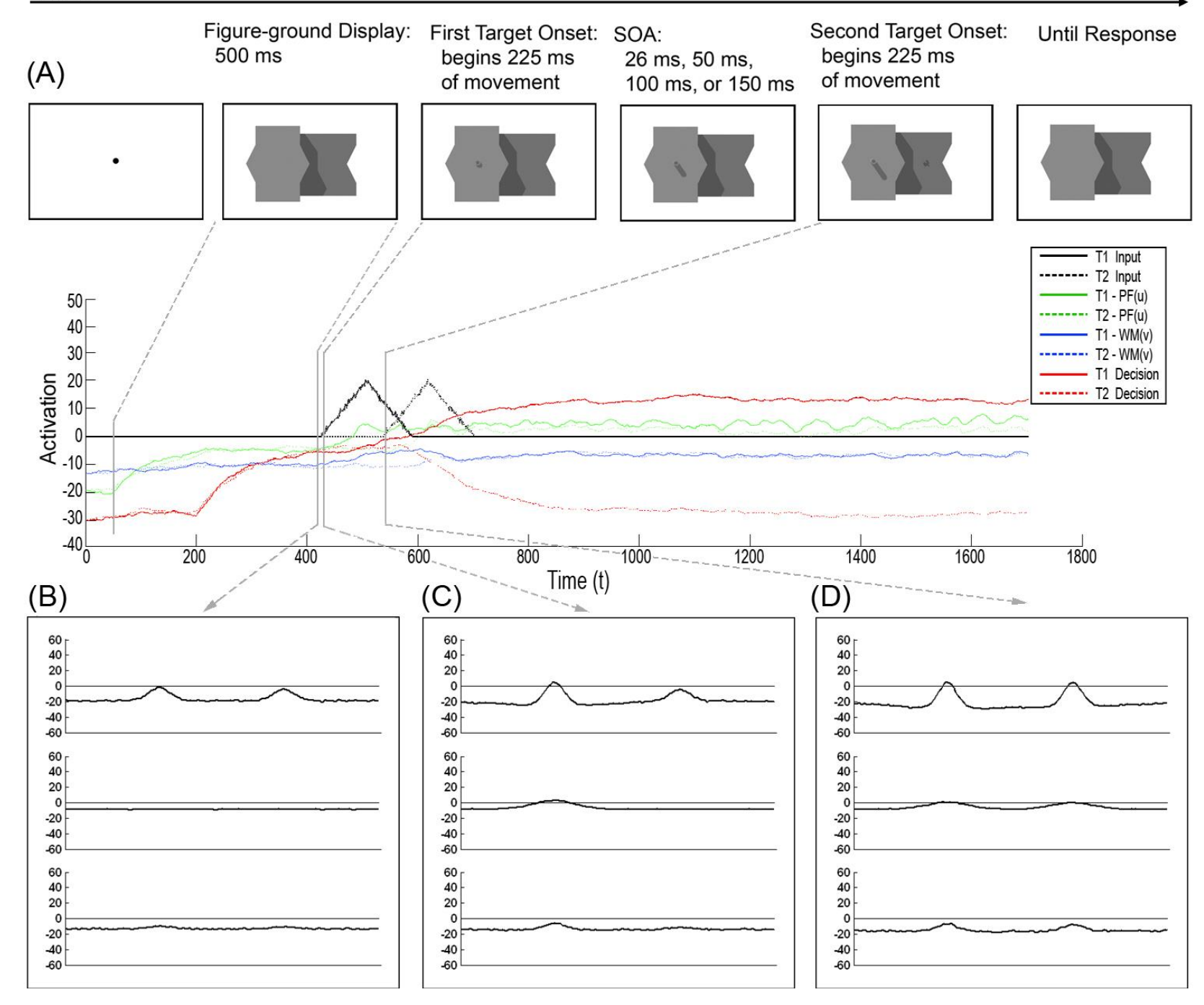

Figure 3. Depiction of a single onset trial presented to the model during the simulations and the three-layer model's activation over time. (A) The progression of trial events over time as presented to the participants and the corresponding activation in the model appears in the panel below. Activation is plotted along the y-axis, with time plotted on the x-axis. Starting at the top of the $y$-axis, the black lines crossing the $y$-axis at 0 indicate the Target input over time. Going down the $\mathrm{y}$-axis, the blue lines indicate activation in the WM field for T1 (figure) and for T2 (ground). The green lines indicate activation in PF for T1 and T2, and the red lines indicating activation in the response field. For all lines, the solid lines indicate activation in response to T1 and the dotted lines are activation levels in response to T2. (B) Activation at the end of the presentation of the figure-ground display prior to the targets' appearances. (C) Activation in PF is higher for the figure location (left) at the end of the initial presentation of the figure target and the SOA (150 ms). (D) Activation levels after the onset of T2. 


\section{Time}
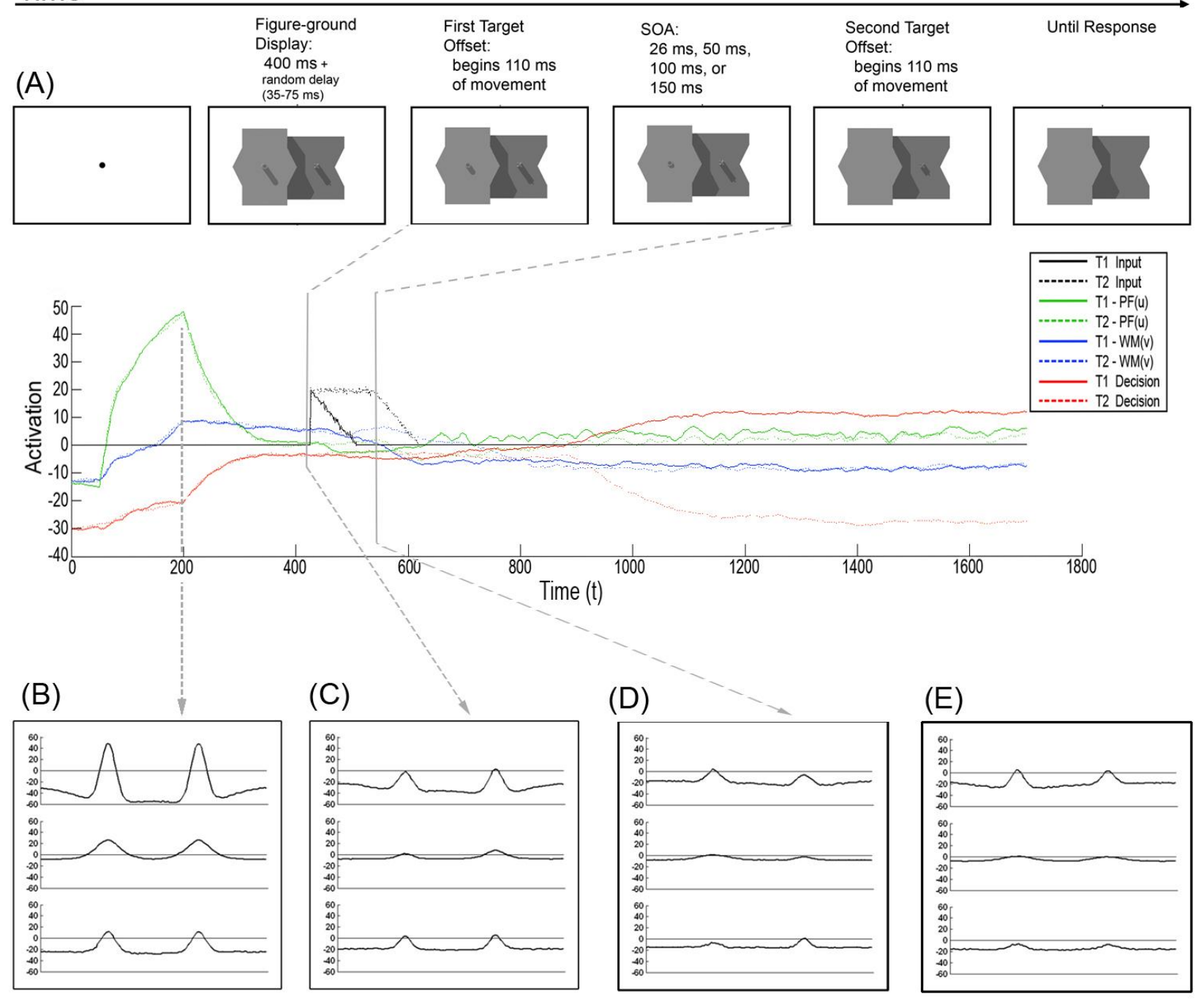

Figure 4. Depiction of a single offset trial presented to the model during the simulations and the three-layer model's activation over time. (A) The progression of trial events over time as presented to the participants and the corresponding activation in the model appears in the panel below. Activation is plotted along the y-axis, with time plotted on the x-axis. Starting at the top of the $y$-axis, the black lines crossing the $y$-axis at 0 indicate the Target input over time. Going down the $\mathrm{y}$-axis, the blue lines indicate activation in the WM field for T1 (figure) and for T2 (ground). The green lines indicate activation in PF for T1 and T2, and the red lines indicate activation in the response field. For all lines, the solid lines indicate activation in response to T1 and the dotted lines are activation levels in response to T2. (B) Activation in the three-layer model at the end of the initial boost with the figure-ground display. (C) Activation at the end of the presentation of the figure-ground display prior to the targets' disappearances. (D) Activation in PF is higher for the figure location (left) at the end of the initial offset of the figure target and the SOA $(150 \mathrm{~ms})$. Note that activation in WM is below threshold for the left (figure) location. (E) Activation levels after the offset of T2. Now both peaks in WM are below threshold. 
(A) Onset Simulations

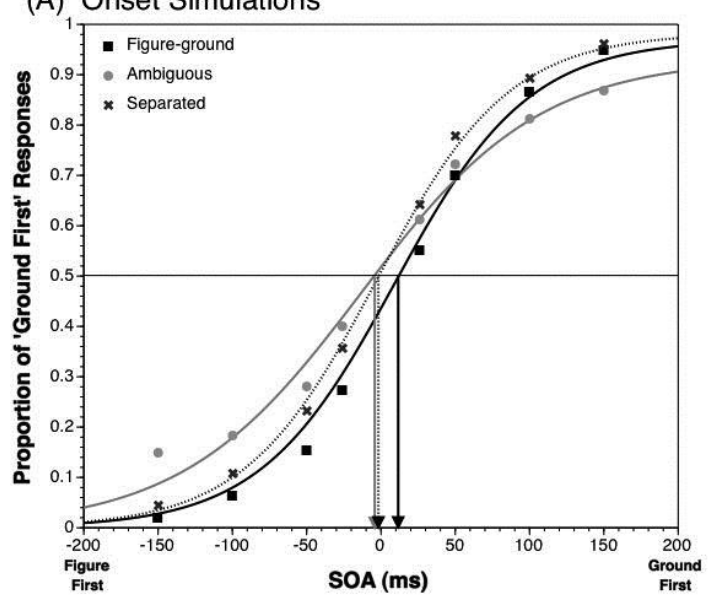

(B) Offset Simulations

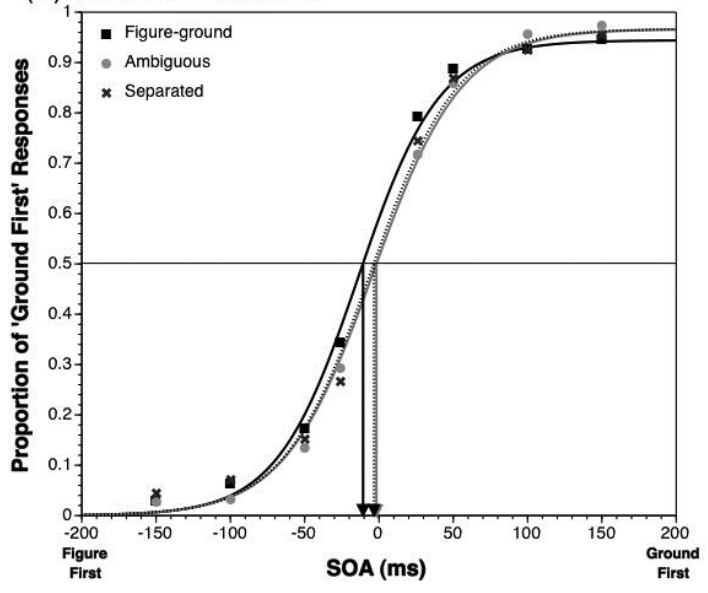

Empirical Data: 'Which onset first?'

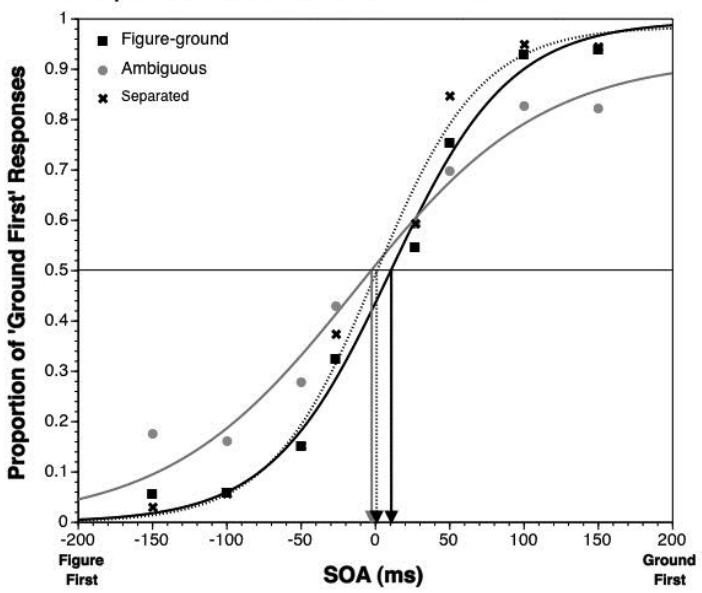

Empirical Data: 'Which offset first?'

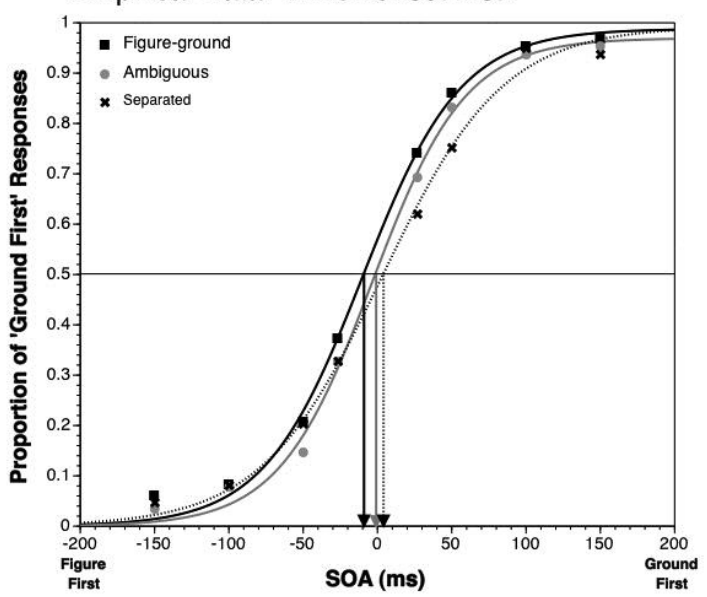

Figure 5. Model simulations (left panels) and empirical experiments (right panels) data for both the (A) onset tasks and the (B) offset tasks. 
(A) Onset Simulations

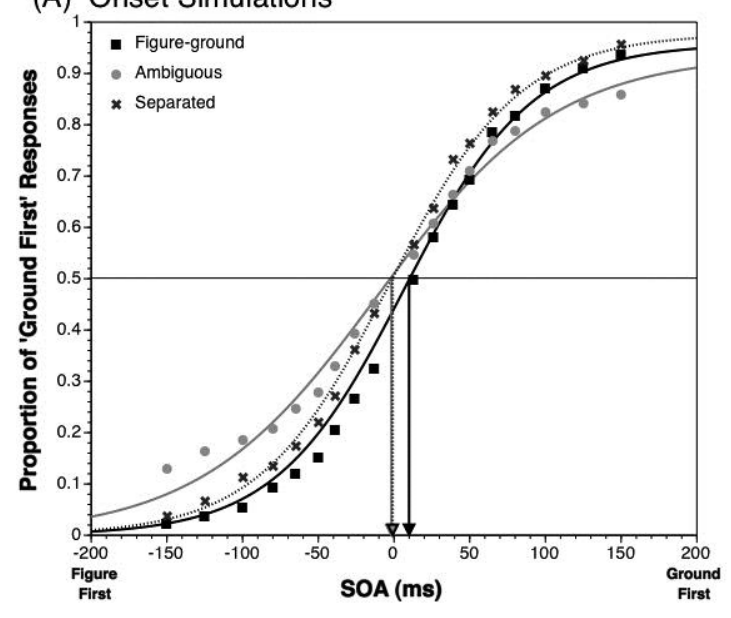

(B) Offset Simulations

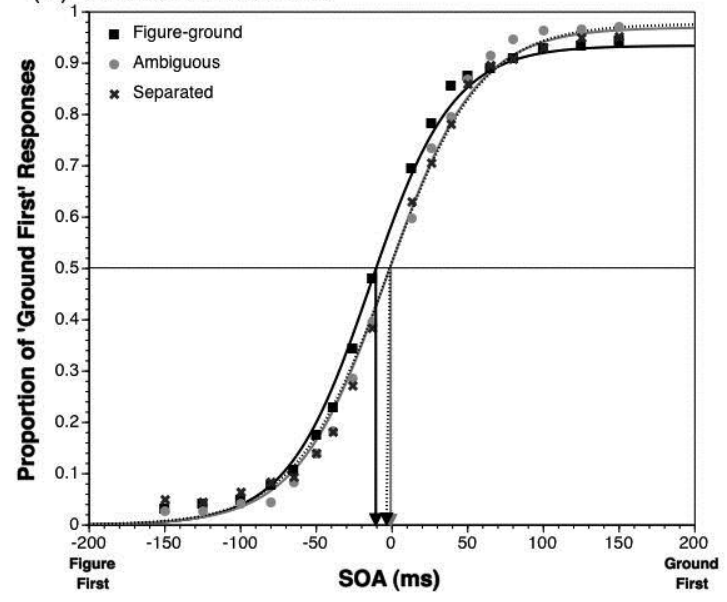

Empirical Data: 'Which onset first?'

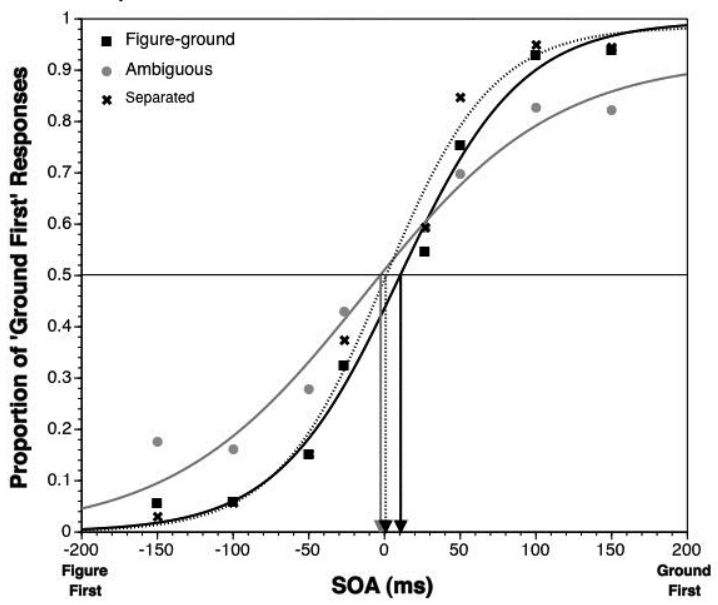

Empirical Data: 'Which offset first?'

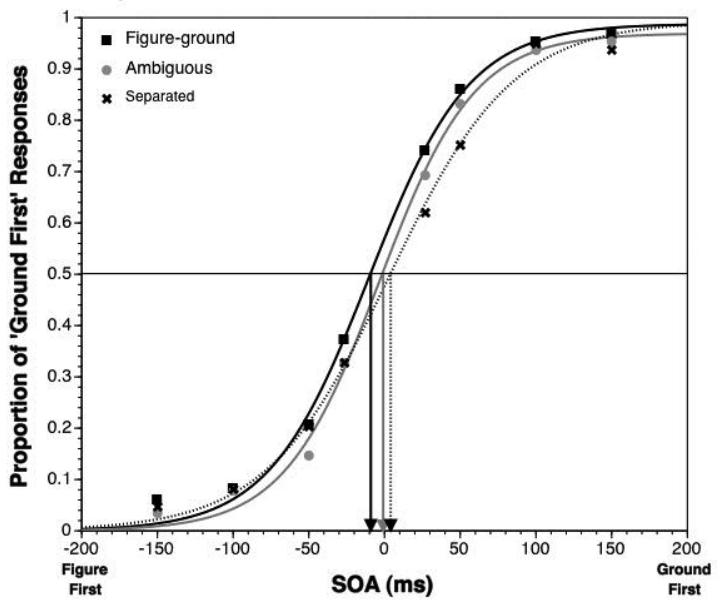

Figure 6. Model simulations (left panels) with a denser sampling across SOAs and empirical experiments (right panels) data for both the (A) onset tasks and the (B) offset tasks. 


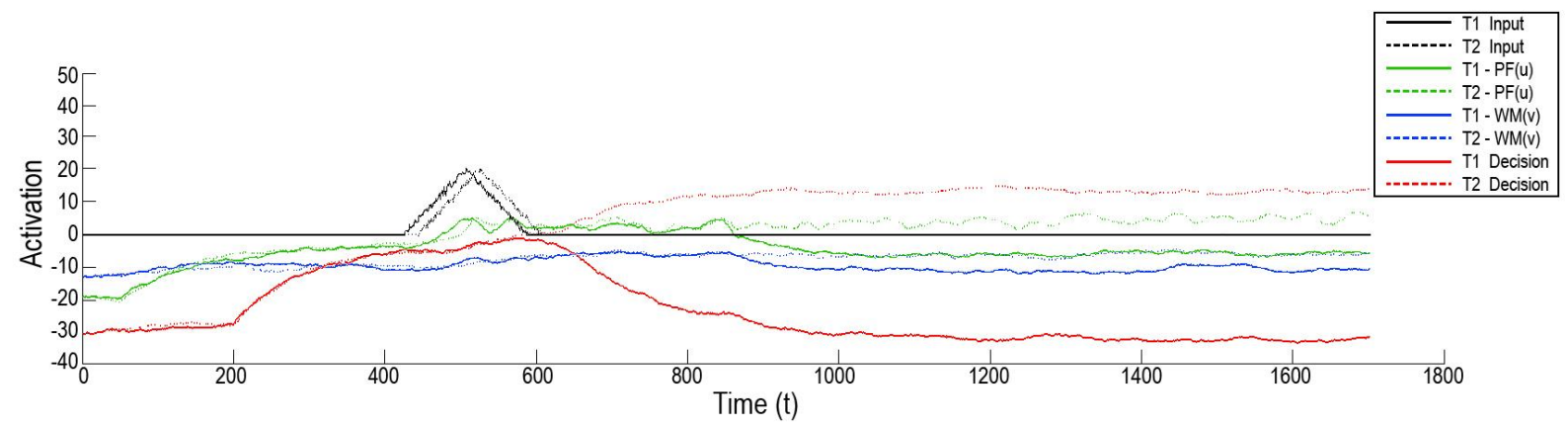

Figure 7. Sample of model simulation's activation (y-axis) over time (x-axis) for a figureground trial in the onset task where the ground target (T1) led by $26 \mathrm{~ms}$. This example demonstrates the model incorrectly responding 'figure' (T2). 


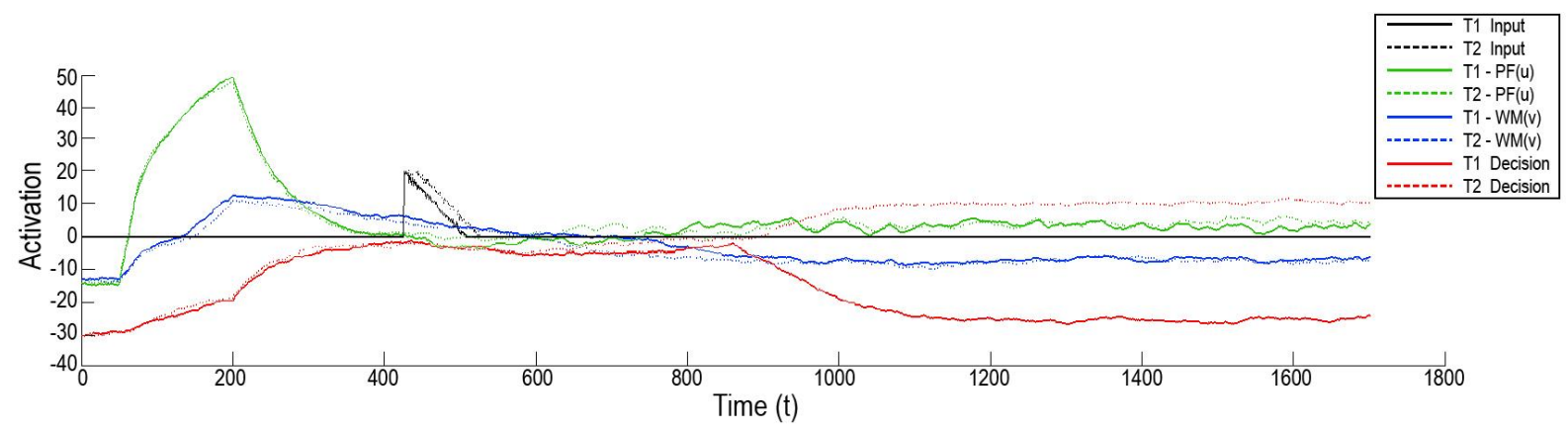

Figure 8. Sample of model simulation's activation (y-axis) over time (x-axis) for a figureground trial in the offset task where the figure target led by $26 \mathrm{~ms}$. This example demonstrates the model incorrectly responding 'ground'. 